\title{
Illicit ketamine induced frequency of micturition in a young Malay female
}

${ }^{1}$ M. H. R. Noorzurani, M. Med (Family Medicine), PhD ${ }^{2}$ Balasingam Vicknasingam, PhD., ${ }^{3}$ Suresh Narayanan, PhD.

${ }^{1}$ Associate Professor, Faculty of Medicine, University Malaya, 50603, Kuala Lumpur, Malaysia

${ }^{2}$ Senior Lecturer, Centre for Drug Research, University Sains Malaysia, 11800 Penang, Malaysia

${ }^{3}$ Professor, School of Social Sciences, University Sains Malaysia, 11800 Penang, Malaysia

\section{Corresponding author:}

Noorzurani, Md Haris Robson., MBBS, M.Med, PhD (Addiction), Associate Professor, Department of Primary Care Medicine, Faculty of Medicine, University of Malaya, 50603, Kuala Lumpur, Malaysia.

Email: noorzurani@um.edu.my

Tel number : 0060172005468

Fax number : 0060379577941

Keywords: ketamine, drug, substance, bladder, cystitis, recreational, club, urinary tract infection, detrusor overactivity 


\section{Abstract}

Issues: The growing use of illicit ketamine among young adults poses a public health concern in Malaysia. In contrast to medical use of ketamine, illicit use has been reported to produce adverse effects on the urinary tract and, in particular the bladder. Approach and key Findings: This case report describes a young female Malay who developed severe bladder symptoms (urinary frequency) after consuming illicit ketamine. A history of illicit ketamine use was initially missed and she was consequently diagnosed and treated for a urinary tract infection. Her symptoms persisted despite several courses of antibiotics. The patient was subsequently referred to an urologist where cystoscopic examination and biopsy of the bladder found changes consistent with interstitial cystitis. This was despite the absence of haematuria. Renal function was normal. Implication: The patient's ketamine use was the most likely cause for the urinary symptoms and bladder changes. Conclusion: Illicit ketamine use may lead to severe urinary tract problems that can be irreversible even after ketamine has ceased. Early diagnosis is critical though difficult when ketamine use is not divulged during the consultation with the physician. Physician consideration of this new clinical entity is important, particularly in cases where the symptoms do not resolve with usual treatment.

Word count: 203 


\section{Introduction}

The use of illicit street ketamine as a recreational drug by youths has been widely reported [1-5]. Ketamine hydrochloride is an N-methyl-D-aspartic acid receptor antagonist commonly prescribed as an anaesthetic agent for paediatric anaesthesia, conscious sedation and anaesthesia in asthmatics. Ketamine causes altered sensations, out of body experiences and euphoric rushes [4]. Physiologically ketamine has been reported to affect the cardiovascular system by increasing heart rate, the respiratory system by increasing respiratory rates, the gastrointestinal system by causing nausea and vomiting and the central nervous system by causing convulsions and hallucinations [6]. Recreational ketamine users have reported 'visual hallucinations' and neardeath experiences $[4,7]$. Illicit ketamine has also been associated with ulcerative cystitis $[1,8$ 10] although no cases have previously been reported in Malaysia.

\section{Case report}

Miss P, a 23 year old single Malay female, who lived with her mother, presented to a hospital clinic with an 11 month history of urinary frequency and urgency. She had consulted four general practitioners (GPs) prior to attending the clinic. All of them had treated her condition as a urinary tract infection and she had subsequently been prescribed several different courses of antibiotics. None of the antibiotics alleviated her symptoms.

She had increased urinary frequency (about 2-3 times per hour) and was forced to wear adult diapers at night to avoid soiling her bed. She did not complain of dysuria, haematuria, fever or loin to groin pain. She had not passed stones in the urine. She frequented recreational centres 
(discotheques and pubs) about 3-4 times per week. These late night outings were reduced after she developed her urinary problems. She has been smoking since age 15 and has been consuming alcohol since age 14. She currently binges alcohol at a rate of 6-10 units/per night on weekends. She remains sexually active, though her symptoms were not found to be due to sexually transmitted diseases. Her past medical and surgical histories were otherwise unremarkable.

She had been taking ketamine for a year prior to presentation. It first started with 'having fun' at the discotheques or pub about once a week; after 2-3 months she described feeling dependent on ketamine and commenced daily consumption. Initially, a packet of ketamine costing Ringgit Malaysia (RM) 200 (USD1= RM3.6) lasted for around 6-8 doses, but just prior to presentation she was using RM 200-300 per day. With ketamine she described feeling relaxed and happy and being in a 'different world'. The effects lasted about 15-20 minutes after snorting the ketamine while the euphoria lasted for up to an hour.

Physical examination was unremarkable. Vital signs were normal and cardiovascular, respiratory, neurological, musculoskeletal and abdominal examinations were also normal. There was no suprapubic tenderness. The bladder and kidneys were not palpable. Urine microscopy was normal and urine culture and sensitivity produced no bacterial growth. The urine was also negative for acid fast bacilli. Haematology revealed normal serum creatinine. The patient was referred to an urologist for assessment.

Extensive investigations were undertaken including bladder cystoscopy which revealed a contracted and inflamed bladder. Biopsy showed histological changes consistent with interstitial cystitis. Ultrasound examination revealed normal kidneys. 
Despite advice the patient continues to use ketamine, although less frequently. Her bladder problems persist, requiring her to wear adult diapers daily.

\section{Discussion}

This case report describes an association of lower urinary tract symptoms, with the illicit use of ketamine. Although cases have been reported in Hong Kong, UK and Canada, to our knowledge, this is the first case reported in Malaysia [1, 2, 9].

Ketamine hydrochloride has been used as an anaesthetic agent in human and veterinary procedures [11]. Similar symptoms have not been reported in patients who have been legally prescribed ketamine for medical purposes. This may be due to the shorter duration of ketamine administration. In recent years its use as a recreational drug has been reported to be associated with severe dysuria, urinary frequency, urgency and gross haematuria $[1,8,10]$. Our patient, after taking ketamine for a month, reported frequency and urgency, but none of the other symptoms. The irritative bladder symptoms usually begin after onset of daily ketamine use and do not respond to antibiotic or steroid therapy. Although symptoms are more common after chronic recreational ketamine use, reports of bladder dysfunction has also been reported as early as few days after ketamine abuse [12]. Other studies report patients have contracted bladders with marked histological inflammatory changes $[2,10]$. The contracted bladder is thought to be responsible for the urinary frequency $[1,2,10]$. Although the exact cause of the syndrome has not been established, it has been suggested that the bladder dysfunction could be induced by direct effect of the drug or its metabolites, or an immunological reaction to the contaminants present in the illicit ketamine [13]. 
The results of urine microscopic examination and cultures of our patient were normal and biopsy of the bladder showed changes consistent with interstitial cystitis. Similar findings have been reported elsewhere [1]. However, some patients in that study were also found to have hydronephrosis, papillary necrosis, raised serum creatinine level and haematuria. Fortunately, our patient did not have haematuria and her renal function was normal. This indicates that ketamine associated cystitis can occur in the absence of advanced kidney disease and haematuria.

The role of illicit street ketamine in inducing irritative bladder symptoms warrants further attention and evaluation. The mechanism by which ketamine induces these pathology changes needs further investigation

With the popularity of ketamine among youths [1-5], it is imperative that physicians be aware of the potential bladder sequelae related to ketamine abuse. Urinary tract problems may persist even after ketamine use has ceased. Early diagnosis is critical but often made difficult as recreational use of ketamine is illegal and patients tend to conceal their drug history. Heightened awareness of this clinical entity should enable physicians to be more proactive in considering ketamine abuse in the differential diagnosis. Furthermore, in a younger patient, diagnosis should be considered where urine culture is negative and the problem does not resolve with antibiotics.

Ketamine users should be counselled to stop use to prevent further disease progression and they may also require rehabilitation beyond conventional counselling. Unfortunately programmes especially tailored for recreational ketamine users are largely unavailable, although programs are being developed in the U.K [10]. The growing use of illicit ketamine not only poses a public health concern but also raises new challenges for the rehabilitation of those who are dependent on this substance. 
Conflict of interest: The authors have been invited to give seminars on drug addiction sponsored by Schering Plough (M) Sdn Bhd. 


\section{Reference:}

1. Chu, P.S., et al., The destruction of the lower urinary tract by ketamine abuse: a new syndrome? BJU Int, 2008. 102(11): p. 1616-22.

2. Shahani, R., et al., Ketamine-associated ulcerative cystitis: a new clinical entity. Urology, 2007. 69(5): p. 810-2.

3. Degenhardt, L., J. Copeland, and P. Dillon, Recent trends in the use of "club drugs": an Australian review. Subst Use Misuse, 2005. 40(9-10): p. 1241-56.

4. Muetzelfeldt, L., et al., Journey through the K-hole: phenomenological aspects of ketamine use. Drug Alcohol Depend, 2008. 95(3): p. 219-29.

5. Loxton, N.J., et al., Impulsivity in Hong Kong-Chinese club-drug users. Drug Alcohol Depend, 2008. 95(1-2): p. 81-9.

6. Smith, K.M., L.L. Larive, and F. Romanelli, Club drugs: methylenedioxymethamphetamine, flunitrazepam, ketamine hydrochloride, and gamma-hydroxybutyrate. American Journal of Health-System Pharmacy, 2002. 59(11): p. 1067-76.

7. Copeland, J., Dillon, P, The health and psycho-social consequences of ketamine use. International Journal of Drug Policy., 2005: p. 122-131.

8. Chen, K.T., N.P. Foo, and H.J. Lin, Frequent visits with urinary symptoms: subtle signs of ketamine abuse. Am J Emerg Med, 2008. 26(9): p. 1061-2.

9. Hoskins, R., Ketamine associated cystitis - a case report. Int Emerg Nurs, 2009. 17(1): p. 69-71.

10. Cottrell, A., et al., The destruction of the lower urinary tract by ketamine abuse: a new syndrome? BJU Int, 2008. 102(9): p. 1178-9; author reply 1179.

11. Trujillo, K.A., J.J. Zamora, and K.P. Warmoth, Increased response to ketamine following treatment at long intervals: implications for intermittent use. Biol Psychiatry, 2008. 63(2): p. 178-83.

12. Huang, Y., Jeng, CM., Cheng, TC. , Ketamine associated ulcerative cystitis. Tzu Chi Med J 2008. 20: p. 44-146.

13. Selby, N., Anderson, J., Bungay, P., Chesterton, LJ., Kolhe, NV., Obstructive nephropathy and kidney injury associated with ketamine abuse. Nephrology Dialysis Transplantation Plus, 2008. 5: p. 310-312. 